\title{
Benzobisthiazoles Represent a Novel Scaffold for Kinase Inhibitors of CLK Family Members
}

\author{
Krisna Prak, ${ }^{\dagger}$ Janos Kriston-Vizi, ${ }^{\dagger}$ A. W. Edith Chan, ${ }^{\ddagger}$ Christin Luft, ${ }^{\dagger}$ Joana R. Costa, ${ }^{\dagger}$ Niccolo Pengo, $^{\dagger}$ \\ and Robin Ketteler* ${ }^{\dagger}$
}

${ }^{\dagger}$ MRC Laboratory for Molecular Cell Biology, University College London, London WC1E 6BT, U.K.

${ }^{\ddagger}$ Wolfson Institute for Biomedical Research, University College London, London WC1E 6BT, U.K.

Supporting Information

ABSTRACT: Protein kinases are essential regulators of most cellular processes and are involved in the etiology and progression of multiple diseases. The cdc2-like kinases (CLKs) have been linked to various neurodegenerative disorders, metabolic regulation, and virus infection, and the kinases have been recognized as potential drug targets. Here, we have developed a screening workflow for the identification of potent CLK2 inhibitors and identified compounds with a novel chemical scaffold structure, the benzobisthiazoles, that has not been previously reported for kinase inhibitors. We propose models for binding of these compounds to CLK family proteins and key residues in CLK2 that are important for the compound interactions and the kinase activity. We identified structural elements within the benzobisthiazole that determine CLK2 and CLK3

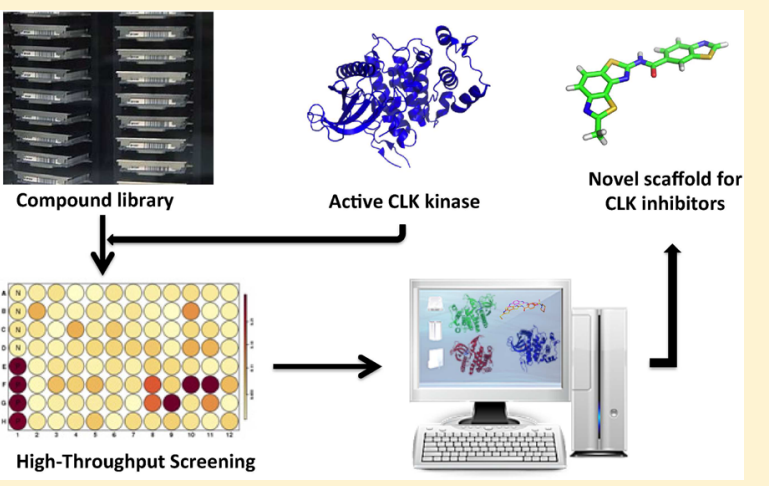
inhibition, thus providing a rationale for selectivity assays. In summary, our results will inform structure-based design of CLK family inhibitors based on the novel benzobisthiazole scaffold.

$\mathrm{P}$ rotein kinases control and modulate a wide variety of biological processes through their catalytic activity, 1,2 including signal transduction and gene splicing. Gene splicing is controlled by the splicing machinery and the assembly of a spliceosome. Spliceosome assembly is mediated by multiple splicing factors, including the hnRNP family of proteins and the serine and arginine rich (SR) proteins. ${ }^{3}$ A key event is the phosphorylation of SR proteins that leads to their nuclear translocation and therefore activation. Kinases that mediate this phosphorylation event are members of the SRPK (SR protein kinase) family, members of the CLK (cdc2-like kinase) family, and topoisomerase 1. The CLKs are dual-specificity kinases (tyrosine and serine/threonine kinases) of the CMGC family (cyclin-dependent kinases, mitogen-activated kinases, glycogen synthase kinases, and CDK-like kinases). There are four mammalian isoforms, CLK1-4, with different substrate targets. CLK activity is controlled by autophosphorylation on multiple serine residues ${ }^{4}$ and phosphorylation at serine 34 , threonine 127, and threonine 344 by AKT $1 / 2 .^{5}$ Members of the CLK family of proteins, most notably, CLK1 and CLK2, are promising drug targets in a variety of diseases. For instance, it has been observed that CLK2 is an insulin-regulated suppressor of hepatic gluconeogenesis. ${ }^{6}$ Additionally, it suppresses hepatic fatty acid oxidation and ketogenesis. ${ }^{7}$ It has also been proposed that CLK2 is a potential drug target for Alzheimer's disease, ${ }^{8,9}$ Gaucher's disease, ${ }^{10}$ and a form of mental retardation, ataxia and atrophy of the brain. ${ }^{11}$ More recently, it has been shown that CLK regulates HIV virus production by modulating splicing of the provirus and affecting gene expression of viral genes. ${ }^{12}$ CLK1 inhibitors are also effective in interfering with influenza virus infection, ${ }^{13}$ suggesting a more general role for CLK inhibitors as antiviral agents. Therefore, there is a demand for developing CLK inhibitors and activators on various disease settings. A number of clinical trials aiming to modify alternative splicing in diverse diseases such as diabetes, acute myeloid leukemia, HIV infection, and cystic fibrosis (see www.clinicaltrials.gov) are ongoing or have been completed. To date, several small molecules targeting SR proteins, topoisomerase I, and kinase inhibitors for SRPKs and CLKs have been developed ${ }^{14}$ (summarized in ref 15). However, most identified inhibitors are potent inhibitors of multiple CLK family members with strongest inhibition of CLK1. Structure-activity relationship studies may aid in the design of more selective compounds.

Here, we studied structural determinants in CLK2 that contribute to ATP binding and catalytic activity to rationalize compound binding. We conducted small molecule screening for the identification of novel CLK2 inhibitors and investigated the selectivity of the compounds on the basis of the kinase crystal structures, the molecular docking, and kinase inhibition assays. Interestingly, we identified a class of benzobisthiazole

Received: October 16, 2015

Revised: December 17, 2015

Published: December 23, 2015 
derivatives, a novel chemical scaffold structure, that exhibit specific profiles among members of the CLK family.

\section{EXPERIMENTAL PROCEDURES}

Plasmid Cloning. cDNA encoding the CLK2 catalytic domain (CLK2cd, amino acids 140-496) was obtained by polymerase chain reaction (PCR) using full-length human CLK2 as a template and BamHI-CLK2 and CLK2-NotI as primers (Supplementary Table 1). The PCR fragment was inserted into bacterial expression vector pGEX6P-1 (GE Healthcare, catalog no. 27-4597-01) using BamHI and NotI, in-frame with an N-terminal GST tag creating pGEXCLK2cd. To generate the CLK2cdK193A (pGEXCLK2cdK193A) and CLK2cdD290A (pGEXCLK2cdD290A) mutants, pGEXCLK2cd was used as a template and two pairs of primers, CLK2-K193A-R and CLK2-K193A-F and CLK2D290A-R and CLK2-D290A-F, were used. All PCRs were conducted for 30 cycles using Pyrobest DNA polymerase (Takara, R005A). To create double mutant CLK2cdK193A/ D290A, pGEXCLK2cdK193A and pGEXCLK2cdD290A plasmids were digested with $N c o$ I and NotI and the resulting longer and shorter DNA fragments from digestion of pGEXCK2cdK193A and pGEXCLK2cdD290A, respectively, were ligated, creating pGEXCLK2cdK193A/D290A. All the clones were verified by DNA sequencing.

Protein Expression and Purification. Expression plasmids pGEXCLK2cd, pGEXCLK2cdK193A, and pGEXCLK2cdK193A/D290A were transformed into Escherichia coli BL21(DE3)-R3-lambda-PPase obtained from the Structural Genomics Consortium (Oxford University, Oxford, U.K.). The proteins were induced by $0.1 \mathrm{mM}$ isopropyl $\beta$-D-1thiogalactopyranoside (IPTG) at $18{ }^{\circ} \mathrm{C}$ overnight. GST-tagged proteins were purified using Glutathione Sepharose 4B (GE Healthcare, catalog no. 17-0756-01) eluted with $50 \mathrm{mM}$ Tris$\mathrm{HCl}$ ( $\mathrm{pH} 8.0$ ), $150 \mathrm{mM} \mathrm{NaCl}, 0.5$ mM EDTA, 0.1 mM EGTA, $10 \mathrm{mM}$ reduced glutathione, and $1 \mathrm{mM}$ DTT. The proteins were further purified by using Amicon ultracentrifugal filter units ultra-15, with a $30 \mathrm{kDa}$ molecular weight cutoff (SigmaAldrich, catalog no. Z717185-8EA), and stored at $-80^{\circ} \mathrm{C}$ in 50 $\mathrm{mM}$ Tris- $\mathrm{HCl}$ (pH 8.0), $150 \mathrm{mM} \mathrm{NaCl}, 0.5 \mathrm{mM}$ EDTA, 0.1 mM EGTA, 33\% glycerol, and 1 mM DTT.

Protein concentrations were determined with a sodium dodecyl sulfate (SDS)-polyacrylamide gel (4 to $20 \%$ gradient, Bio-Rad, catalog no. 456-1096) electrophoresis of the purified proteins and quantification of the Coomassie-stained gel using ImageJ protein quantification (http://openwetware.org/wiki/ Protein Quantification Using ImageJ), with bovine serum albumin (BSA) (abcam, ab102536) as a standard. Three different amounts of proteins were loaded on each gel, and the total intensity of each band corresponding to the purified enzyme was calculated by comparing the band intensity to the corresponding BSA standard curve.

Analysis of Enzyme Kinetics for CLK2cd and Its Mutants. Purified GST-CLK2cd, GST-CLK2cdK193A, and GST-CLK2cdK193A/D290A at 14.5, 72.46, and $362.32 \mathrm{nM}$, respectively, were incubated with 2 -fold serial dilutions of a mixture of ATP (Sigma, catalog no. A7699) and S6K peptide (KRRRLASLR) (SignalChem, catalog no. S05-58) in a 1:5 ratio (ATP:S6K) from 20 to $0.15 \mu \mathrm{M}$ in $10 \mu \mathrm{L}$ of kinase assay buffer A $[25 \mathrm{mM}$ Tris-HCl (pH 7.5), $0.1 \mathrm{mg} / \mathrm{mL}$ BSA, $10 \mathrm{mM}$ $\mathrm{MgCl}_{2}, 0.5 \mathrm{mM} \mathrm{NaO}_{3} \mathrm{~V}, 5 \mathrm{mM} \beta$-glycerophosphate, and $2 \mathrm{mM}$ DTT] in white 384-well plates (Greiner bio-one, catalog no. $781904)$ for $1 \mathrm{~h}$ at $30{ }^{\circ} \mathrm{C}$. Ten microliters of Kinase-Glo
(Promega, catalog no. V6713) was then added to the reaction mixture and incubated for $5 \mathrm{~min}$ at room temperature before the luminescence was read in the PerkinElmer Envision II instrument. The initial velocity (molar per second, $y$ axis) was defined as the change in the concentration of ATP used for phosphorylated S6K, which was plotted versus the concentration of substrate ATP before reaction (molar, $x$ axis). The curves were then fitted using the nonlinear regression method in R software, from which the $V_{\max }$ and $K_{\mathrm{m}}$ (Michaelis constant) for each enzyme-substrate reaction were derived. The $k_{\text {cat }}$ (catalytic constant) was determined by dividing $V_{\max }$ by the enzyme concentration. The catalytic efficiency is defined as $k_{\text {cat }} /$ $K_{\mathrm{m}}$ (inverse molar liter per second).

High-Throughput Screening. A small molecule inhibitor library consisting of 1600 compounds was obtained from LIFE Chemicals (Kiev, Ukraine). These compounds are part of the CUTE kinase collection that was predicted by in silico profiling against a panel of targets and includes potent kinase inhibitor compounds. ${ }^{16}$ Compounds were dissolved as $10 \mathrm{mM}$ stocks in DMSO and stored at room temperature, in the presence of $5 \%$ humidity and 5\% oxygen in the dark in a Roylan Storage Pod system. Compounds were dispensed into white 384-well plates using the Labcyte Echo520 instrument at a final concentration of $10 \mu \mathrm{M}$. Next, $5 \mu \mathrm{L}$ of $144.92 \mathrm{nM}$ purified GST-CLK2cd in kinase assay buffer A was added to the compounds, incubated for $10 \mathrm{~min}$ at room temperature prior to addition of $5 \mu \mathrm{L}$ of a 2 $\mu \mathrm{M} \mathrm{ATP} / 10 \mu \mathrm{M}$ S6K peptide mixture, and incubated again for $60 \mathrm{~min}$ at room temperature. Subsequently, $10 \mu \mathrm{L}$ of KinaseGlo was added to the wells, and the plates were analyzed after $10 \mathrm{~min}$ on the Envision II multilabel plate reader (PerkinElmer). TG003 (Sigma, catalog no. T5575) and DMSO were used as a positive control and a negative control, respectively. The percentage of inhibition is equal to $100-$ [(total ATP before assay - remaining ATP in assay with compound)/(total ATP before assay - remaining ATP in assay with DMSO) $\times 100]$. The compounds that gave $\geq 50 \%$ inhibition were selected for $\mathrm{IC}_{50}$ analysis. NR9 was obtained from Enzo Life Sciences (catalog no. ALX-270-463-M001).

For kinase inhibition assays, the final concentrations of the enzymes were $15,14.5,14.5$, and $8.8 \mathrm{nM}$ for CLK1, CLK2, CLK3, and Dyrk1A (Invitrogen, catalog no. PV3785), respectively. The compound concentrations ranged from 15503 to $121 \mathrm{nM}$ and from 1550 to $12 \mathrm{nM}$. The RS domainderived peptide (GRSRSRSRSR) (Anaspec, catalog no. 61722) for CLK1 and CLK3, Dyrktide (RRRFRPASPLRGPPK) (Anaspec, catalog no. 62698) for Dyrk1A, and S6K peptide for CLK2 were used as substrates at a concentration of $5 \mu \mathrm{M}$.

Luciferase Inhibition Assay. For analysis of luciferase inhibition, $10 \mu \mathrm{L}$ of luciferase reagent (Kinase-Glo, Promega) was added to $10 \mu \mathrm{L}$ of a mixture consisting of $1 \mu \mathrm{M}$ ATP and 5 $\mu \mathrm{M}$ S6K and $10 \mu \mathrm{M}$ compound (potential kinase inhibitor) in buffer $\mathrm{A}$ in the absence of kinase and incubated for $10 \mathrm{~min}$ at room temperature in a white well plate before the luminescence was read. The percentage of inhibition is equal to (luminescence in the assay without compound - luminescence in the assay with compound)/(luminescence in the assay without compound) $\times 100$.

Kinase Profiling. 3A5 and 5E4 were sent to the International Centre for Kinase Profiling at the MRC Protein Phosphorylation and Ubiquitylation Unit for profiling (as described in ref 17). Compounds were profiled against 140 kinases using a radioactive $\left(\left[{ }^{33} \mathrm{P}\right] \mathrm{ATP}\right)$ filter binding assay at a 


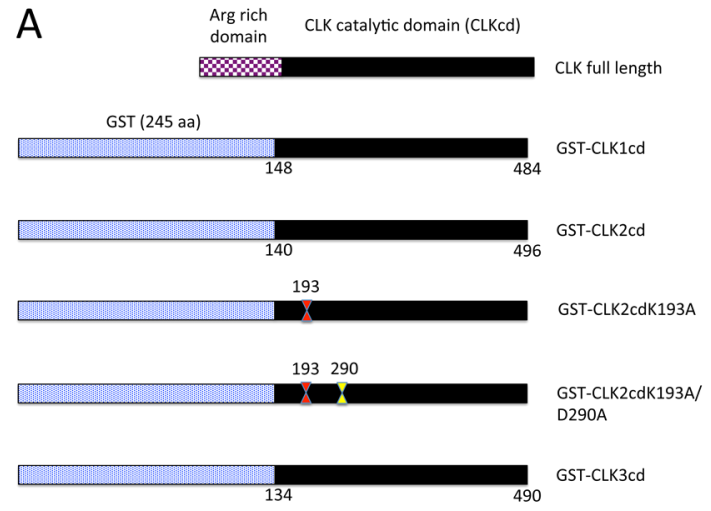

B
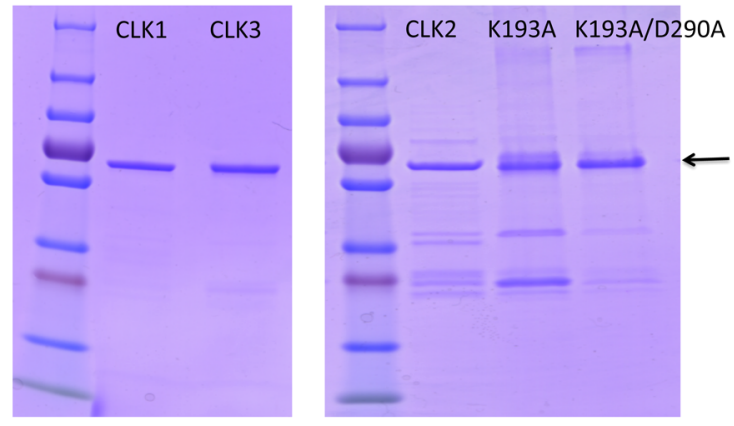

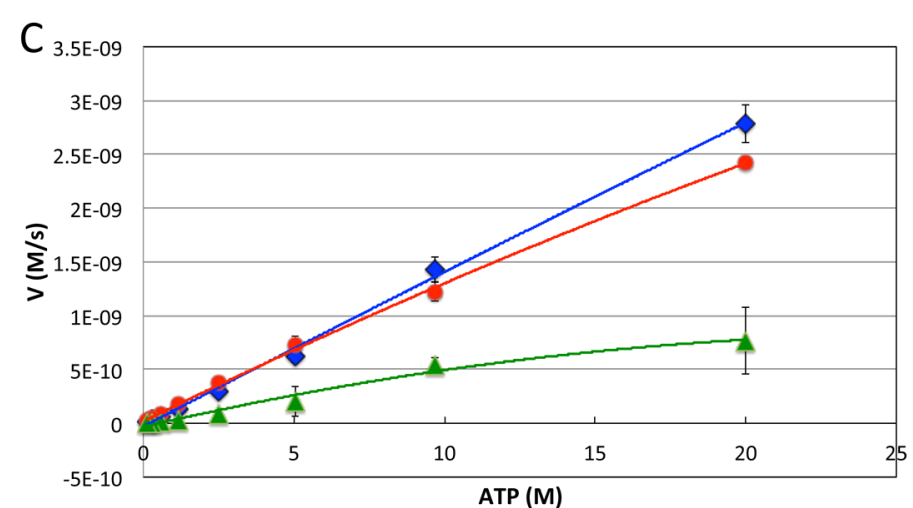

D

\begin{tabular}{lcccc} 
& \multicolumn{4}{c}{ Catalytic kinetics of CLK2 and its mutants } \\
\cline { 2 - 5 } & Km (M) & Kcat $(1 / \mathrm{s})$ & Kcat/Km (1/M.s) & $\begin{array}{c}\text { Catalytic activity } \\
\text { compared to CLK2cd }\end{array}$ \\
\hline GST-CLK2cd & $312.7 \pm 23.9$ & 4.48 & $143.2 \mathrm{E}-04$ & 1 \\
\hline GST-CLK2cdK193A & $46.3 \pm 3.1$ & 0.13 & $27.8 \mathrm{E}-04$ & 0.194 \\
\hline $\begin{array}{l}\text { GST-CLK2cdK193A/ } \\
\text { D290A }\end{array}$ & $14.8 \pm 2.4$ & 0.0038 & $2.6 \mathrm{E}-04$ & 0.018 \\
\hline
\end{tabular}

Figure 1. Plasmid construction, protein purification, and catalytic activity analysis of CLK and its mutants. (A) Schematic diagram of GST-CLKcd (GST-tagged CLK catalytic domain) and GST-CLK2cd mutants used in this study. The arginine rich domain in wild-type CLK2 is depicted as a checkered box and the catalytic domain (cd) as black box. Residues 193 and 290 in the catalytic domain of CLK2 are highlighted. (B) Purification of GST-CLKcd and GST-CLK2cd mutants. The catalytic domains of CLK1, CLK2, CLK3, CLK2K193A, and CLK2K193A/D290A were tagged with GST, subjected to SDS-PAGE, and stained with Coomassie brilliant blue. The arrow indicates the bands corresponding to $1 \mu \mathrm{g}$ of the purified enzymes. (C) Catalytic activities of CLK2 and mutants. Blue diamonds, red circles, and green triangles depict data for GST-CLK2cd, GSTCLK2cdK193A, and GST-CLK2cdK193A/D290A, respectively. The catalytic activity was determined after incubation of the purified kinase with substrate at $30^{\circ} \mathrm{C}$ as described in Experimental Procedures. The initial velocity ( $y$ axis) was defined as the change in the concentration of ATP used for phosphorylated S6K, which was plotted vs the concentration of substrate ATP before reaction ( $x$ axis). (D) Enzyme kinetics of GST-CLK2cd and its mutants were determined as described for panel $\mathrm{C}$ and quantified.

concentration of $1 \mu \mathrm{M}$. Results were obtained as inhibition of kinase activity and are listed in Supplementary Table 2.

Molecular Docking. In silico docking of kinase inhibitors and ATP into the ATP binding pocket of CLK2 \{human X-ray structure [Protein Data Bank (PDB) entry 3NR9] cocrystallized with ligand (5Z)-5-(quinolin-6-ylmethylidene)-2-[(thiophen-2-ylmethyl)amino]-1,3-thiazol-4(5H)-one (ligand code, NR9) $\}$ and CLK3 (human CLK3 X-ray structure (PDB entry 2WU6) cocrystallized with ligand 5-amino-3-\{[4(aminosulfonyl)phenyl]amino $\}-N$-(2,6-difluorophenyl)- $1 H$ 1,2,4-triazole-1-carbothioamide (ligand code, DKI)) was performed using GOLD. ${ }^{18,19}$ The binding site used in the docking study was defined by residues within $6 \AA$ of the cocrystallized ligand. Molecules 3A5, 5E4, 5F4, and NR9 were docked against 3NR9 and 2WU6 using GOLD. The four lowest conformations of each molecule were generated by MOE [Molecular Operating Environment (MOE), 2014.09, Chemical Computing Group Inc., Montreal, QC] as initial structures for GOLD docking. GOLD docking was performed using GOLD's genetic algorithm (GA) with scoring function ChemScore $^{20}$ as it is more suitable for the kinase family. The three best binding poses from each inhibitor, determined by their fitness scores, were saved and examined visually for both CLK2 and CLK3. The cocrystallized ligand (NR9-3NR9 and DKI-2WU6) molecules were docked back into the protein structures as a validation test. In all the docking poses for 3NR9
(CLK2) and 2WU6 (CLK3), GOLD correctly predicted the binding conformation as that in the $\mathrm{X}$-ray crystallographic structure.

\section{RESULTS}

Residues K193 and D290 in CLK2 Are Required for Catalytic Activity. To identify small molecule inhibitors of the CLK family of proteins, we developed an in vitro kinase assay for CLK2 that is amenable to high-throughput drug screening. We generated N-terminal GST fusions of the wild-type (WT) CLK2 catalytic domain (GST-CLK2cd) and mutant forms that interfere with ATP binding or catalytic activity (GSTCLK2cdK193A or GST-CLK2cdK193A/D290A, respectively) (Figure 1A), expressed them in E. coli BL21(DE3)-R3-lambdaPPase, and purified them using Glutathione Sepharose 4B (Figure 1B). To measure the activities of WT CLK2 and CLK2 mutants, we determined the conversion of ATP substrate at different concentrations at $30{ }^{\circ} \mathrm{C}$ for $1 \mathrm{~h}$. GST-CLK2cdK193A/ D290A and GST-CLK2cdK193A showed a substrate conversion significantly slower than that of GST-CLK2cd (Figure 1C). We calculated the catalytic rates of the enzyme and the mutants (Figure 1D) and found that the $K_{\text {cat }} / K_{\mathrm{m}}$ values of GST-CLK2cdK193A/D290A, GST-CLK2cdK193A, and GSTCLK2cd were $2.6 \times 10^{-4}, 27.8 \times 10^{-4}$, and $143.2 \times 10^{-4} \mathrm{M}^{-1}$ $\mathrm{s}^{-1}$, respectively, which indicated that the K193A mutation or K193A/D290A double mutation leads to the catalytic activity 

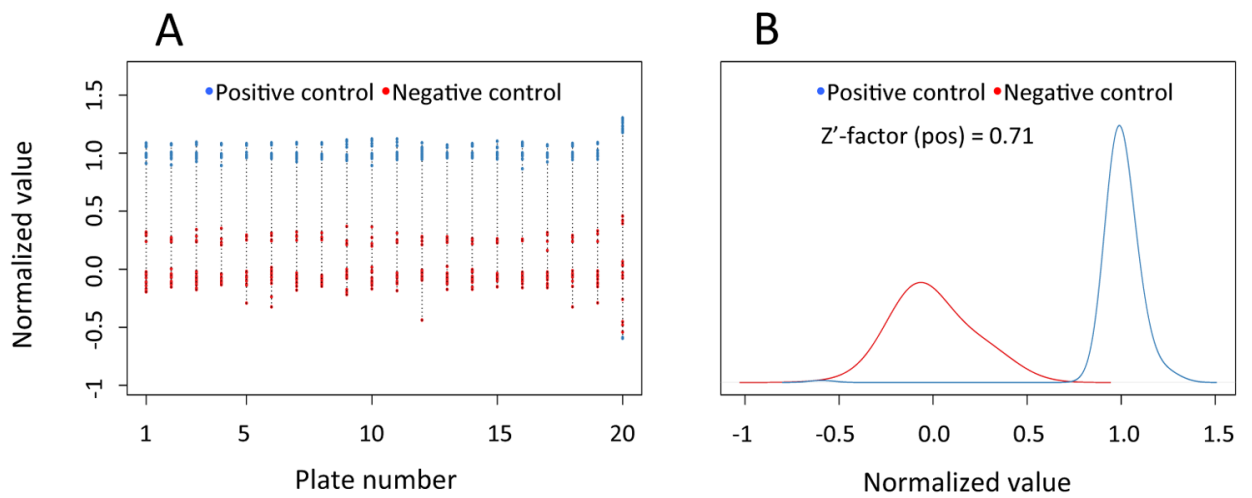

C
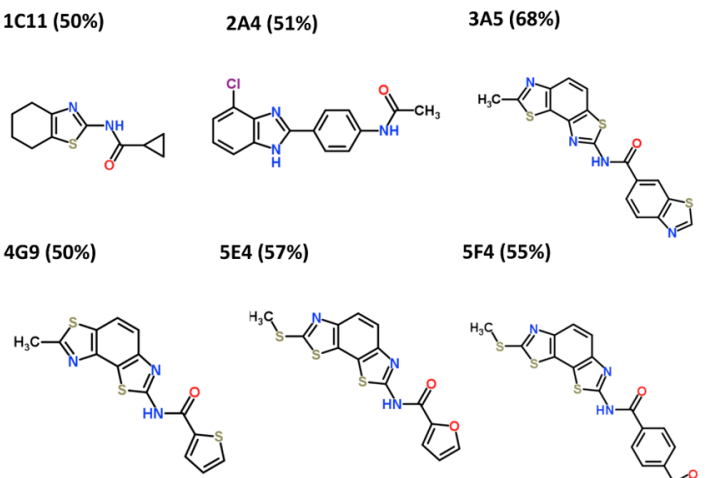

$5 E 4$ (57\%)

5F4 (55\%)

$3 B 5$ (65\%)

4B9 (56\%)<smiles>CC(C)(C)Nc1nc2cc3c(cc2s1)OCC3</smiles>

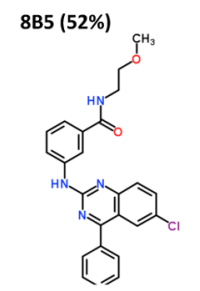

$9 \mathrm{C6}(58 \%)$

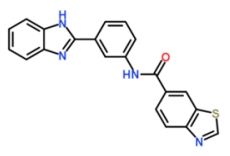

$5 E 11(58 \%)$<smiles>Fc1ccc(-c2csc3ncnc(Cc4ccccc4)c23)cc1</smiles>

$7 C 4$ (53\%)

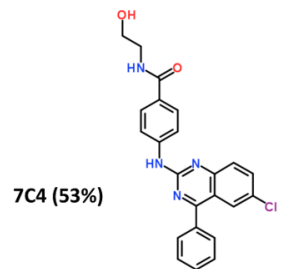

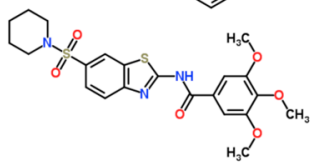

13D3 (51\%)

$17 F 6$ (53\%)

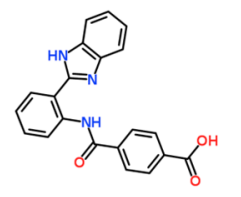

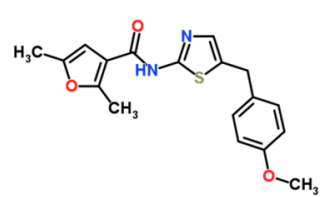

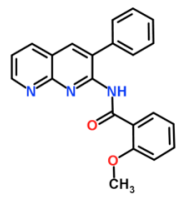

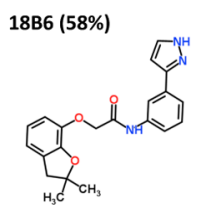

18H3 (56\%)

TG003 (63\%)
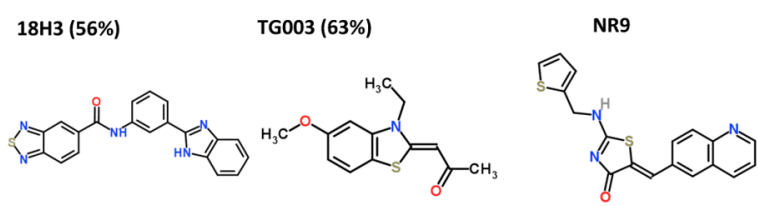

D<smiles>c1nc2c(ccc3nc[se]c32)[se]1</smiles>

benzo[1,2-d:3,4']bisthiazole

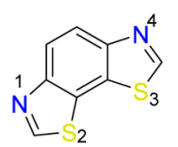

benzo[1,2-d:4,3']bisthiazole

Figure 2. High-throughput screening (HTS) for CLK2 inhibitors. (A) Normalized screening data for 1600 compounds from a LIFE Chemicals library. Each plate contains 80 compounds in four replicates, 32 replicates for substrate (ATP+S6K) only, and 16 replicates each for positive and negative control samples. The cellHTS $2^{30}$ software package (R, Bioconductor) was used to process the data. Raw data points were normalized with the normalized percent inhibition (NPI) method. (B) Kernel density estimate functions were calculated from the normalized positive and negative control values, and a $Z^{\prime}$ factor was calculated using the cellHTS2 Bioconductor package. (C) Chemical structures of TG003 and the hit compounds. The percentage of inhibition of the compounds at $10 \mu \mathrm{M}$ on CLK2 is shown in parentheses. (D) Chemical structure of the two isomeric benzobisthiazole scaffolds.

of CLK2 being decreased $>5$ - or $>54$-fold, respectively. We therefore propose that both K193 and D290 contribute to the catalytic activity of CLK2.

Identification of Small Molecule Inhibitors of CLK2. Next, we screened a small molecule inhibitor library consisting of 1600 compounds from LIFE Chemicals that were predicted to be kinase inhibitors by in silico profiling. ${ }^{16}$ The screening was conducted at a final concentration of $10 \mu \mathrm{M}$ in quadruplicate.
Using TG003, a known inhibitor of CLK1 and CLK2 activity ${ }^{21}$ as a positive control, we measured the conversion of ATP using a luciferase-based in vitro kinase assay as described in Experimental Procedures. Comparing TG003 inhibition to that of DMSO-treated controls, we determined a $Z$ prime factor of 0.71 (Figure 2A,B), which we found suitable for highthroughput screening. 
Table 1. Inhibition of Luciferase by Hit Compounds at $10 \mu \mathrm{M}^{a}$

\begin{tabular}{|c|c|c|c|c|c|c|c|c|c|}
\hline & TG003 & $1 \mathrm{C} 11$ & $2 \mathrm{~A} 4$ & $3 \mathrm{~A} 5$ & $3 \mathrm{~B} 5$ & $4 \mathrm{~B} 9$ & $4 \mathrm{G} 9$ & $5 \mathrm{E} 4$ & $5 \mathrm{E} 11$ \\
\hline$\%$ inhibition & $13.4 \pm 1.8$ & $8.1 \pm 1.8$ & $17.5 \pm 4.5$ & $6.7 \pm 2.8$ & $26 \pm 1.1$ & $20.4 \pm 1.6$ & $47.8 \pm 2.1$ & $29.7 \pm 2.9$ & $26.4 \pm 2.4$ \\
\hline & $5 F 4$ & 7C4 & $8 \mathrm{~A} 6$ & $8 \mathrm{~B} 5$ & 9C6 & 13D3 & 17F6 & 18B6 & $18 \mathrm{H} 3$ \\
\hline$\%$ inhibition & $23.6 \pm 1.5$ & $19.6 \pm 3.6$ & $18.8 \pm 1.6$ & $0 \pm 6.2$ & $5.6 \pm 4.0$ & $0 \pm 2.4$ & $21.9 \pm 0.2$ & $17.5 \pm 2.2$ & $21.1 \pm 2.2$ \\
\hline
\end{tabular}

${ }^{a}$ Luciferase inhibition was measured by combining $10 \mu \mathrm{L}$ of luciferase reagent with $10 \mu \mathrm{L}$ of a mixture consisting of $1 \mu \mathrm{M}$ ATP and $5 \mu \mathrm{M} \mathrm{S} 6 \mathrm{~K}$ and $10 \mu \mathrm{M}$ compound (potential kinase inhibitor) in reaction buffer after incubation for $10 \mathrm{~min}$ at room temperature. The percentage of inhibition is equal to (luminescence in the assay without compound - luminescence in the assay with compound)/(luminescence in the assay without compound) $\times 100$.

Hits were initially ranked by percentage of inhibition compared to DMSO control. Seventeen hits were identified as potential inhibitors of CLK2 at $\geq 50 \%$ inhibition in the primary screen (Figure 2C). Among the hit compounds, four benzobisthiazoles were identified (3A5, 4G9, 5E4, and 5F4). Because some compounds may reduce luciferase activity or elicit autoluminescence, we tested all hits for potential interference with luciferase activity. Compounds were incubated with firefly luciferase in the absence of kinase, and the percentage of inhibition of luciferase activity was measured (Table 1). Most compounds did not significantly reduce or increase luciferase activity, with the exception of benzobisthiazole 4G9 that reduced luciferase activity by $47.8 \pm 2.1 \%$. We are therefore unable to assess the CLK2 inhibition for 4G9 with the assay used. The other three benzobisthiazoles (3A5, 5E4, and 5F4) were then taken forward for further studies as potential inhibitors of CLK2 activity (Figure 2D). To determine the $\mathrm{IC}_{50}$ values for TG003 and benzobisthiazoles $3 \mathrm{~A} 5,5 \mathrm{E} 4$, and 5F4, we assayed dose-response curves of the compounds from 15503 to $121 \mathrm{nM}$ and from 1550 to $12 \mathrm{nM}$. The $\mathrm{IC}_{50}$ values for CLK2 inhibition by TG003, 3A5, 5E4, and 5F4 were determined to be $353,68,174$, and $127 \mathrm{nM}$, respectively (Table 2). In conclusion, compounds 3A5, 5E4, and $5 \mathrm{~F} 4$ are potent inhibitors of CLK2 activity with $\mathrm{IC}_{50}$ values similar to or slightly better than that of known inhibitor TG003.

Table 2. $\mathrm{IC}_{50}$ Values for Inhibitors for CLK Family Members $^{a}$

$\begin{array}{llllll} & \text { TG003 } & \text { NR9 } & \text { 3A5 } & \text { 5E4 } & \text { 5F4 } \\ \text { CLK1 (nM) } & 49 & 390 & 51 & 277 & 273 \\ \text { CLK2 (nM) } & 353 & 553 & 68 & 174 & 127 \\ \text { CLK3 (nM) } & >3000 & >3000 & 346 & >3000 & >3000 \\ \text { DYRK1A (nM) } & 204 & 596 & 260 & 334 & 213\end{array}$

${ }^{a} \mathrm{IC}_{50}$ values were determined for the five inhibitors for CLK1, CLK2, CLK3, and DYRK1A. Literature values for TG003 according to Muraki et al.: ${ }^{21}$ CLK1, $20 \mathrm{nM}$; CLK2, $200 \mathrm{nM}$; CLK3, >10 $\mu \mathrm{M}$; CLK4, $15 \mathrm{nM}$. Literature values for TG003 according to Fedorov et al.: ${ }^{14}$ CLK1, $48.6 \mathrm{nM}$; CLK3, >4 $\mu \mathrm{M}$; Dyrk1A, $156.1 \mathrm{nM}$. Literature values for TG003 according to Mott et al.: ${ }^{26}$ CLK1, 19 nM; CLK2, 95 $\mathrm{nM}$; CLK3, $3 \mu \mathrm{M}$; CLK4, $30 \mathrm{nM}$; Dyrk1A, $12 \mathrm{nM}$.

Kinase Profiling. To determine the specificity for the novel CLK2 inhibitors, we profiled compounds 3A5 [ $N$-(2-methyl$[1,3]$ thiazolo $[5,4-e][1,3]$ benzothiazol-7-yl)-1,3-benzothiazole6-carboxamide $]$ and 5E4 $\{N$-[7-(methylsulfanyl) $[1,3]$ thiazolo$[4,5-g][1,3]$ benzothiazol-2-yl]-2-furamide $\}$ against a Premier Screen-kinase panel (Supplementary Table 2) (http://www. kinase-screen.mrc.ac.uk/premier-screen). ${ }^{17}$ The panel used consists of 140 kinases (138 human kinases, one rat kinase, and one Tribolium castaneum kinase) and covers the whole breadth of potential kinases. Compounds 5E4 and 3A5 were confirmed as very potent inhibitors of CLK2 with 97\% inhibition at $1 \mu \mathrm{M}$. Furthermore, both compounds displayed a strong inhibitory activity toward the related kinase DYRK1A (97\% for 5E4 and $84 \%$ for 3A5) and some inhibitory activity toward other kinases (see Supplementary Table 2). Other CLK family members were not presented in the panel. Overall, these compounds display high potency toward the CLK family of kinases.

Inhibitor Binding and Specificity against CLKs and Dyrk1A. Next, we were interested in assessing the selectivity of the benzobisthiazoles toward other CLK family members and the closely related DYRK1A kinase. We analyzed the inhibitory effect of 3A5, 5E4, 5F4, and two known inhibitors of CLK2 (NR9 and TG003) against CLK1, CLK2, CLK3, and Dyrk1A (Supplementary Figure S1). We determined the $\mathrm{IC}_{50}$ values for TG003 to be 49, 353, >3000, and $204 \mathrm{nM}$ for CLK1, CLK2, CLK3, and Dyrk1A, respectively (Table 2). Compounds 5E4 and 5F4 were equally effective inhibitors against the tested kinases. Among the tested compounds, 3A5 was the most potent inhibitor for the kinases with $\mathrm{IC}_{50}$ values of $51,68,346$, and $260 \mathrm{nM}$ for CLK1, CLK2, CLK3, and Dyrk1A, respectively. NR9 had the poorest inhibitory effect against the kinases with an $\mathrm{IC}_{50}$ value of $553 \mathrm{nM}$ against CLK2.

To understand the preference of the compounds in inhibition of CLK1 and CLK2 over other related kinases, we compared the kinase domain structures of CLK1, CLK2, CLK3, and DYRK1A to identify key residues that contribute to catalytic activity. Analysis of the structural superimposition of the protein kinase domains (Figure $3 \mathrm{~A}$ ) revealed root-meansquared ( $\mathrm{rms}$ ) distances of the aligned atoms of $0.598,0.556$, 0.734 , and $>1.2 \AA$ for CLK1 (PDB entry 2VAG) and CLK3 (PDB entry 2WU6), CLK1 and CLK2 (PDB entry 3NR9), CLK2 and CLK3, and Dyrk1A (PDB entry 4MQ1) and CLKs, respectively. These results indicate that the catalytic domain of CLK2 is most similar to that of CLK1. This is in agreement with the $\mathrm{IC}_{50}$ values for the identified inhibitors that target both CLK1 and CLK2 equivocally.

The crystal structure of CLK2 allows the identification of residues that line the ATP binding pocket and may thus contribute to compound binding. Using Pymol software ${ }^{22}$ for surface viewing of the CLK2 structure (PDB entry 3NR9), we identified multiple amino acid residues that contribute to the formation of hydrophilic and hydrophobic patches of the ATP binding pocket (Figures 3 and 4). Structural studies of CLK1 in complex with inhibitors V25 (PDB entry 2VAG) and DBQ (PDB entry 1Z57) allowed us to identify conserved residues that contribute to inhibitor binding, including F172, K191, E292, ${ }^{14,23-25}$ and F241. ${ }^{26}$ Together with CLK2 structural data, this suggests that the corresponding residues F174, K193, F243, and E294 in CLK2 play a significant role in compound binding.

We then examined the binding of the benzobisthiazole moiety in CLK2 by in silico molecular docking. For 3A5, the 

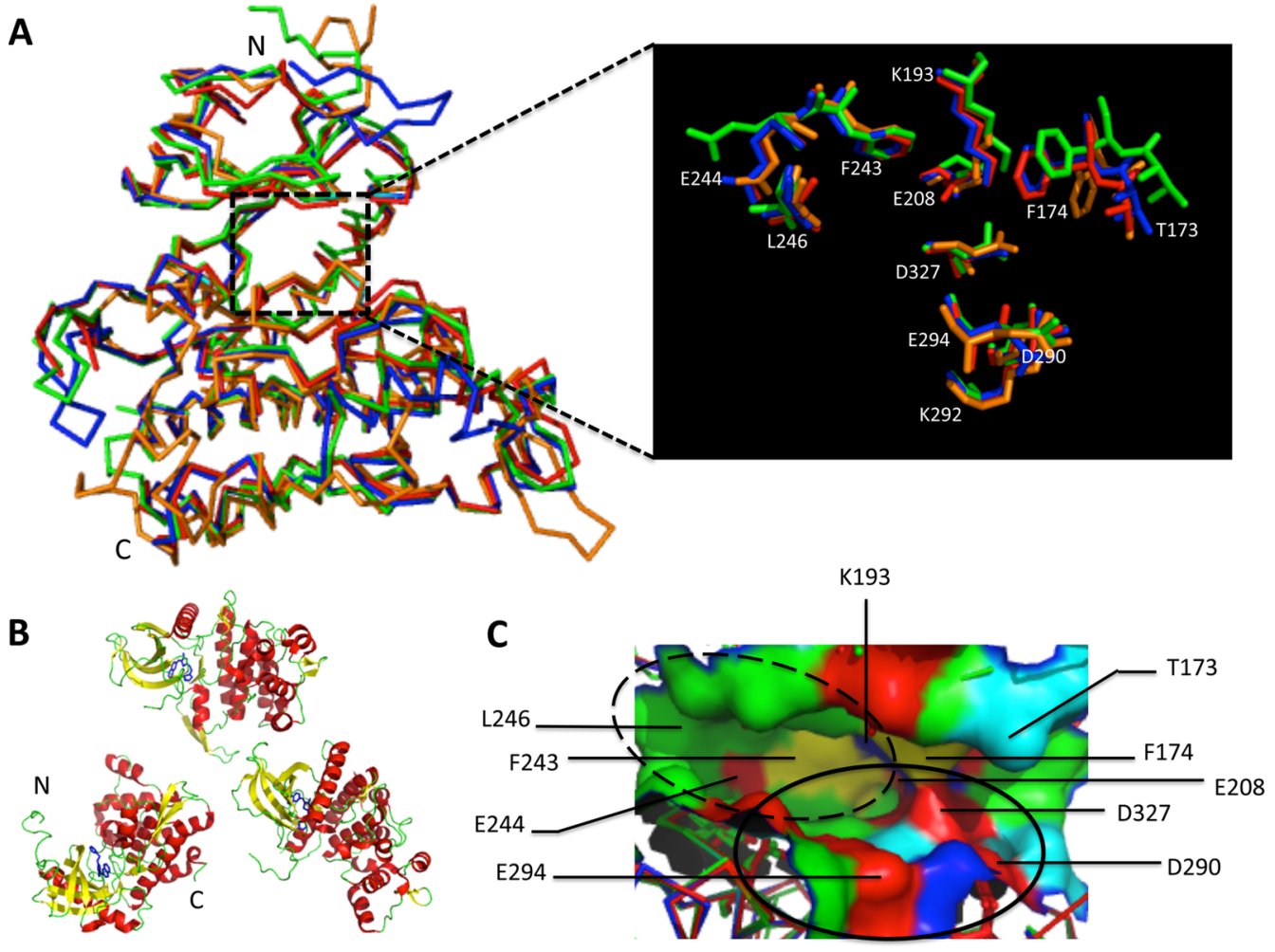

Figure 3. Overview of the CLK2 kinase domain structure. (A) Structural superimposition of CLK1 (PDB entry 2VAG), CLK2 (PDB entry 3NR9), CLK3 (PDB entry 2WU6), and Dyrk1A (PDB entry 4MQ1), colored red, blue, green, and orange, respectively. The dashed squared box indicates the ATP binding pocket of the kinases. (B) Ribbon diagram representation of the CLK2 kinase domain in a trimeric structure. The secondary structures are shown as yellow $\beta$ sheets and red helices, and the NR9 inhibitors are colored blue. (C) Surface of the ATP binding pocket of CLK2 showing negatively charged amino acid residue surfaces in red, positively charged residues in blue, polar residues in cyan, hydrophobic residues in green, and phenylalanine residues in yellow. ATP binding and catalytic sites are denoted by the dashed and solid oval, respectively. Figures were generated using PyMOL. ${ }^{22}$

benzobisthiazole docked into the adenine binding site, with the nitrogen atom in the ring making a hydrogen bond to the backbone $\mathrm{NH}$ group of L246 (Figure 5B) as in the case of NR9. The methyl group of the benzobisthiazole docked into hydrophobic pocket I. The benzothiazole ended up in hydrophobic pocket II. Compared to NR9, it lacks the hydrogen bond formed with K193. However, the extra hydrophobic interaction in pocket II may explain the more potent and specific inhibition of 3A5 over NR9. The structure of 3 A5 contains a benzobisthiazole scaffold and a benzothiazole joined by an amide bond. One could speculate that an inverse binding mode is possible; e.g., the benzothiazole binds in the hinge region. Although the two ring systems are similar, they are quite different in size, the former being a tricyclic ring and the latter a bicyclic. This will have an effect in the docking experiment. In fact, in our docking results, only one of 10 solutions was an inverse binding pose, and the docking score was the worst. This is mainly because the hydrophobic interaction between the protein and the larger benzobisthiazole is weakened.

On the other hand, the inhibitions of 5E4 and 5F4 against CLK2 are decreased $\sim 2-3$-fold compared to that of 3A5. The benzobisthiazole scaffolds of 5E4 and 5F4 are slightly different isomeric types compared to that of 3A5. Some of the relevant heteroatoms are in different positions in these two chemotypes, so it may not be surprising that a different affinity for the enzyme is observed. However, because there is an extra thiomethyl $\left(-\mathrm{SCH}_{3}\right)$ attached to the ring of $5 \mathrm{E} 4$ and $5 \mathrm{~F} 4$, instead of a methyl $\left(-\mathrm{CH}_{3}\right)$ as in $3 \mathrm{~A} 5$, it could also influence the interaction between the molecule and the protein. From the docking result, to balance the hydrophobic interaction with the kinase, 5E4 does not form a hydrogen bond with hinge L246, but instead a hydrogen bond can be formed with the D327 side chain in CLK2 (Figure 5B). 5F4 is slightly larger than 5E4, and the extension of the molecule makes the overall structure less planar. Although 5F4 is predicted to form a hydrogen bond with hinge L246, the carbonyl group $(\mathrm{C}=\mathrm{O})$ seems to have a negative effect on the overall binding as it is not optimally occupying a pocket with the backbone $\mathrm{C}=\mathrm{O}$ group of E294. Thus, the binding mode of 3A5, 5E4, and 5F4 differs considerably, with the benzobisthiazole contributing significantly to the overall kinase binding.

Of the five inhibitors used in this study, only $3 \mathrm{~A} 5$ is a potent inhibitor of CLK3 with an $\mathrm{IC}_{50}$ of $346 \mathrm{nM}$ (Table 2). To rationalize this, the five inhibitors were docked against a structure of CLK3 (PDB entry 2WU6). In the X-ray crystal structure of CLK3, the cocrystallized inhibitor DKI (K00546) forms three bidentate hydrogen bonds with the backbone E237 and L239 (Figure 5D). ${ }^{14}$ The phenylsulfonamide binds in hydrophobic pocket I while the difluorophenyl in hydrophobic pocket II. DKI binds in a DFG-in conformation of classic kinase binding sites. The docking results indicated that only $3 \mathrm{~A} 5$ can form the classic bidentate hydrogen bonding with the backbone L239 (Figure 5C). The benzothiazole occupies hydrophobic region II. Other inhibitors such as TG003, NR9, 5E4, and 5F4 cannot form any hydrogen bond at the hinge region (Figure 


CLK2
CLK3
CLK1
CLK4
DYrk1a

CLK2

CLK3

CLK1

CLK4

Dyrkla

CLK2

CLK3

CLK1

CLK4

Dyrkla

CLK2

CLK3

CLK1

CLK4

Dyrkla

CLK2

CLK3

CLK1

CLK4

Dyrkla

CLK2

CLK3

CLK1

CLK 4

Dyrkla

CLK2

CLK3

CLK1

CLK 4

Dyrkla

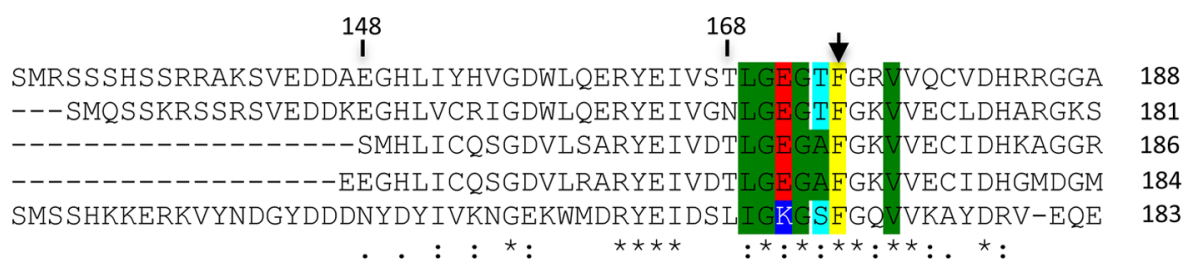

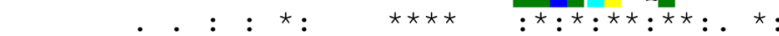
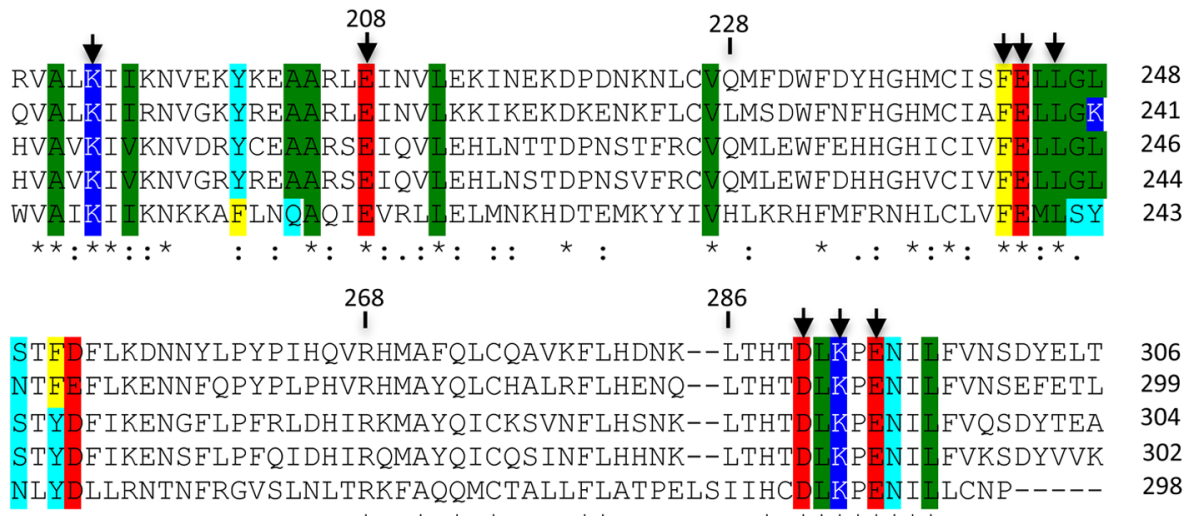

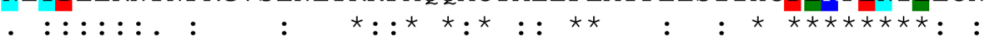

346

YNLEKKRDERSVKSTAVRVVDFGSATFDHEHHSTIVSTRHYRAPEVILELGWSQPCDVWS 366

YNEHKSCEEKSVKNTSIRVADFGSATFDHEHHTTIVATRHYRPPEVILELGWAQPCDVWS 359

YNPKIKRDERTLINPDIKVVDFGSATYDDEHHSTLVSTRHYRAPEVILALGWSQPCDVWS 364

YNSKMKRDERTLKNTDIKVVDFGSATYDDEHHSTLVSTRHYRAPEVILALGWSQPCDVWS 362

---------KRSAIKIVDFGSSCQLGQRIYQYIQSRFYRSPEVLLGMPYDLAIDMWS 346

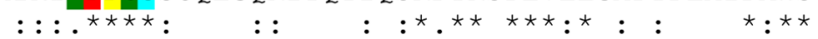

386

IGCI IFEYYVGFTLFQTHDNREHLAMMERI LGP I PSRMIRKTRKQKYFYRGRL--DWDEN

IGCILFEYYRGFTLFQTHENREHLVMMEKI LGP I PSHMI HRTRKQKYFYKGGL--VWDEN

IGCILIEYYLGFTVFPTHDSKEHLAMMERI LGPLPKHMIQKTRKRKYFHHDRL--DWDEH

IGCILIEYYLGFTVFQTHDSKEHLAMMERI LGPI PQHMIQKTRKRKYFHHNQL--DWDEH

LGC I LVEMHTGEPLF SGANEVDQMNK IVEVLGI P PAH I LDQAPKARKFFEKLPDGTWNLK

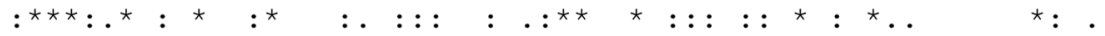

441

TSA-G--RYVRENCKPLRRYLT------------SEAEEHHQLEDLIESMLEYEPAK 466

SSD-G--RYVKENCKPLKSYML--------------QDSLEHVQLFDLMRRMLEFDPAQ 459

SSA-G--RYVSRACKPLKEFML-------------SQDVEHERLFDLIQKMLEYDPAK 464

SSA-G--RYVRRRCKPLKEFML-------------CHDEEHEKLFDLVRRMLEYDPTQ 462

KTKDGKREYKPPGTRKLHNILGVETGGPGGRRAGESGHTVADYLKFKDLILRMLDYDPKT 466

$$
\text { . * . }{ }^{*}: *^{*}: \quad::: *^{*}:{ }^{*}:::^{*}
$$

486

RLTLGEALQHPFFARLRAEPPNKLWDSSRD

RITLAEALLHPFFAGLTPEERSF--HT--- 484

RITLREALKHPFFDLLKKSI-------- 484

RITLDEALOHPFFDLL------------ 478

RIQPYYALQHSFFKKTADE----------- 485

$\star \quad * \star * \star *$

Figure 4. Multiple-sequence alignment of the catalytic domain of human CLK1, CLK2, CLK3, CLK4, and Dyrk1A. Amino acid residues contributing to the surface of the ATP binding pocket are colored as follows: red, blue, cyan, green, and yellow for negatively charged, positively charged, polar amino acid, hydrophobic, and phenylalanine residues, respectively. Arrows indicate amino acid residues that contribute significantly to the formation of the ATP binding pocket of the kinases. The multiple-sequence alignment was prepared by ClustalW2.

$5 \mathrm{E})$. The absence of the bidentate bond may explain the inactivity of these inhibitors toward CLK3.

$3 \mathrm{~A} 5$ had a stronger inhibitory effect against $\mathrm{CLK} 2\left(\mathrm{IC}_{50}=68\right.$ $\mathrm{nM})$ than against CLK3 $\left(\mathrm{IC}_{50}=346 \mathrm{nM}\right)$, though it formed more hydrogen bonds with CLK3 than with CLK2 (panels C and $\mathrm{A}$ of Figure 5). For the investigation, we examined the binding pose of the inhibitor in the kinases (Figure 6). We found that the hydrophobic interaction between $3 \mathrm{AS}$ and
CLK2 was stronger than that between $3 \mathrm{~A} 5$ and CLK3. This phenomenon is well-supported by the similarity of their ATP binding pockets (Figure 3A).

\section{DISCUSSION}

CLK2 has emerged as a promising drug target in a variety of diseases, including neurodegeneration and virus infection. ${ }^{6-8}$ The crystal structure of CLK2 in complex with NR9 is available, 

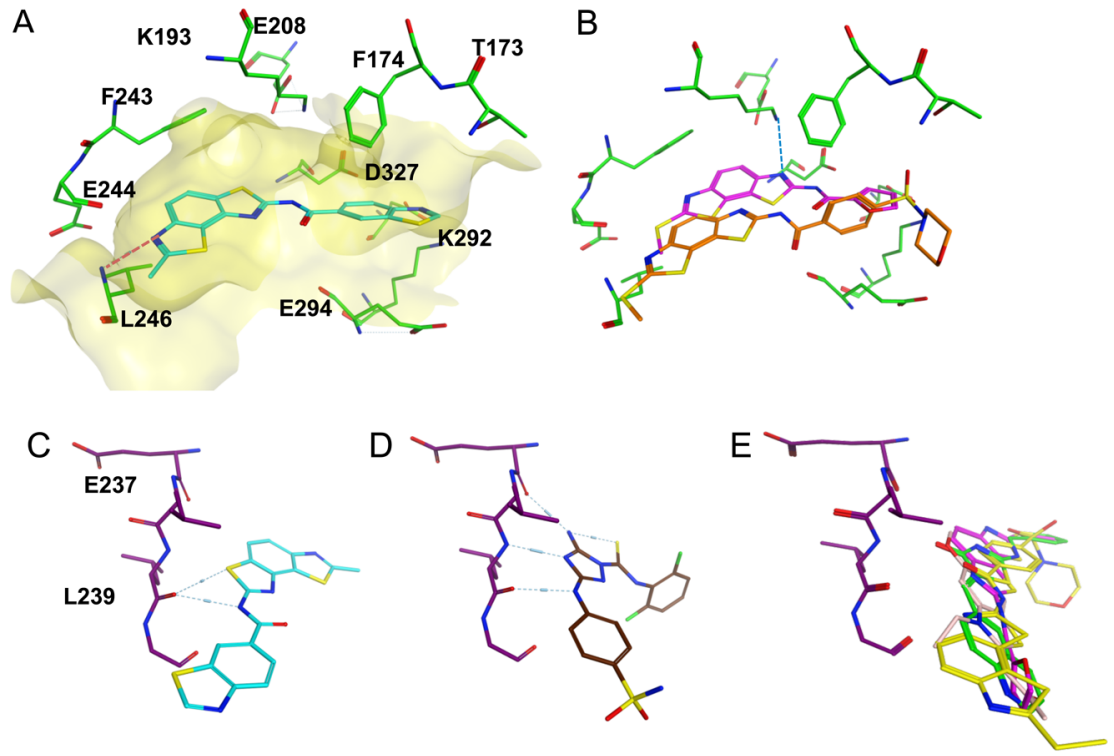

Figure 5. Docking models of small molecules in the ATP binding site of CLK2 and CLK3. (A) Predicted binding pose of 3A5 (C atoms colored cyan) in CLK2. A hydrogen bond is formed between the backbone NH group of L246 and the nitrogen atom in the benzobisthiazole ring. The ATP binding site is shown as a yellow surface. (B) Predicted binding poses for 5E4 (C atoms colored magenta) and 5F4 (C atoms colored orange) in CLK2. (C) Predicted binding pose for 3A5 (C atoms colored cyan) in CLK3 (PDB entry 2WU6, C atoms colored purple). Only the hinge region is shown. (D) Cocrystallized DKI (K00546, C atoms colored brown) in CLK3 (PDB entry 2WU6, C atoms colored purple). (E) Predicted binding pose for 5E4 (C atoms colored magenta), 5F4 (C atoms colored orange), NR9 (C atoms colored green), and TG003 (C atoms colored pink) in CLK3 (PDB entry 2WU6, C atoms colored purple). Only the hinge region is shown.

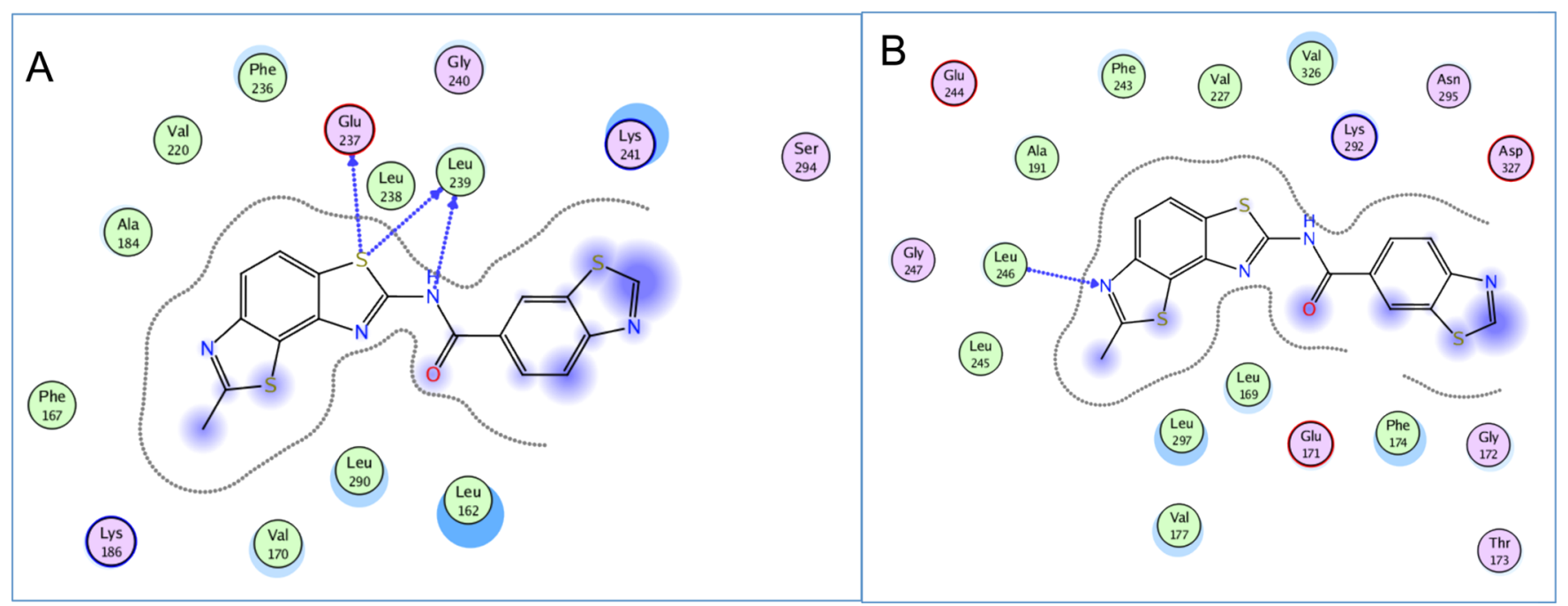

Figure 6. Interaction diagram of 3A5 in the ATP binding site of (A) CLK3 and (B) CLK2. (A) Predicted binding pose of 3A5 in CLK3. There are three H-bonds formed between the ligand and E237, L238, and L239 of CLK3 beside polar interaction with K241, S294, and K186 and hydrophobic interaction with A184, V220, F236, V170, L290, and L162. (B) Predicted binding pose of 3A5 in CLK2. A hydrogen bond is formed between the backbone NH group of L246 and the nitrogen atom in the benzobisthiazole. There are strong hydrophobic interactions between the ligand and L245, L246, A191, F243, V227, V326, V177, L297, L169, and F174 of CLK2 in addition to polar contact between the ligand and E171, K292, and D327.

but no further information regarding how the structure relates to activity has been reported. Here, we employed a small molecule screen, involving 1600 compounds from the CUTE LIFE Chemicals kinase inhibitor library, to identify new potent inhibitors of CLK2. As a result, we identified the benzobisthiazole scaffold as a potent inhibitory structure for CLK2 and other CLK family members. The benzobisthiazole scaffold has previously been reported in novel bacterial helicase inhibitors, ${ }^{27}$ thus showing promise for further drug development. A substructure search of the benzobisthiazole (Figure
2D) moiety in $\mathrm{ChEMBL}^{28}$ revealed that this scaffold is novel among around $54 \mathrm{~K}$ known kinase inhibitors.

The novel benzobisthiazoles displayed remarkable potency in inhibition of CLK1, CLK2, and DYRK1A, supporting the notion that this scaffold is a good starting point for the development of inhibitors for the CLK family of proteins. Interestingly, of the three benzobisthiazoles, only compound 3A5 displayed significant inhibitory activity toward CLK3. We propose that this selectivity is mediated by an interaction of the benzobisthiazole of 3A5 with L239 in the backbone of CLK3, 
thus allowing classic bidentate hydrogen bonding with CLK3, and further modification of the hinge region binding ${ }^{29}$ of $3 \mathrm{AS}$ would enable us to obtain a very potent and selective benzobisthiazole-based inhibitor against CLK3. The underlying difference in binding compared to that of 5E4 and 5F4 is based on the different heteroatom arrangement in the benzobisthiazole scaffolds (see Figures 2 and 3B): The core scaffold of the hits is a tricyclic benzobisthiazole. For 3A5, the most potent inhibitor of CLK2, the two sulfur atoms are in positions 2 and 4 of the ring system (benzo[1,2- $\left.d: 3,4^{\prime}\right]$ bisthiazole). In this case, the two sulfur atoms are not on the same side of the ring system. In 5E4 and 5F4, the two sulfur atoms are in the 2,3 position of the ring system (benzo[1,2-d:4,3'] bisthiazole) and the two sulfur atoms are on the same side of the ring system. Their docking results against CLK2 are quite different. This could be due to the different arrangement of the position of the heteroatoms, the groups attached to the amide linker, the strong hydrophobic pocket of CLK2 toward the compound with a benzobisthiazole scaffold, and the overall shape of the molecule. Similar to this study, a previous study also noted substantial differences in inhibitor binding for CLK1 compared to the structure of CLK3. ${ }^{14}$ This was attributed to a preference for CLK3 to undergo conformational changes with the P loop moving away from the ATP binding site upon inhibitor binding. Similar conformational changes may contribute to differential binding of the benzobisthiazoles to CLK2 and CLK3. Future studies using cocrystallization can prove this theory.

In summary, our study identified a novel chemical motif, the benzobisthiazole, as a selective and potent inhibitor for CLK family members. These studies will be informative in the future for selecting and modeling better compounds to improve selectivity toward the CLK family.

\section{ASSOCIATED CONTENT}

\section{S Supporting Information}

The Supporting Information is available free of charge on the ACS Publications website at DOI: 10.1021/acs.biochem.5b01128.

Supplementary Figure S1 and primers used for plasmid cloning for GST-CLK1cd, GST-CLK2cd, and GSTCLK3cd and site-directed mutagenesis of CLK2cdK193A and CLK2cdK1933/D290A (Supplementary Table 1) (PDF)

Kinase profiling of small compounds $3 \mathrm{~A} 5$ and 5E4 (Supplementary Table 2) (XLSX)

\section{AUTHOR INFORMATION}

\section{Corresponding Author}

*MRC Laboratory for Molecular Cell Biology, University College London, Gower Street, London WC1E 6BT, U.K. Email: r.ketteler@ucl.ac.uk. Telephone: +44(0)2031084063. Fax: +44(0)2076797805.

\section{Funding}

This work was supported by the UK Medical Research Council core funding to the MRC-UCL University Unit (ref. MC_EX_G0800785), BBSRC funding (BB/J015881/1), the Wellcome Trust $(101472 / \mathrm{Z} / 13 / \mathrm{Z})$, and the UCL Capital Investment Fund. The UCL Confidence in Concept scheme funded by the MRC provided funding to support this project (MC_PC_12024). The European Union Seventh Framework
Programme (FP7/2007-2013, grant PIRG08-GA-2010276811) supported J.K.-V.

\section{Notes}

The authors declare no competing financial interest.

\section{ACKNOWLEDGMENTS}

We thank SGC Oxford for E. coli strain BL21(DE3)-R3lambda-PPase and Richard Angell for helpful discussions.

\section{ABBREVIATIONS}

CLK, CDC2-like kinase; HTS, high-throughput screening; CLK1cd, CDC2-like kinase 1 domain; CLK2cd, CDC2-like kinase 2 domain; CLK3cd, CDC2-like kinase 3 domain; GST, glutathione $S$-transferase; WT, wild type; DMSO, dimethyl sulfoxide.

\section{REFERENCES}

(1) Manning, G., Whyte, D. B., Martinez, R., Hunter, T., and Sudarsanam, S. (2002) The protein kinase complement of the human genome. Science 298, 1912-1934.

(2) Yao, Z., Petschnigg, J., Ketteler, R., and Stagljar, I. (2015) Application guide for omics approaches to cell signaling. Nat. Chem. Biol. 11, 387-397.

(3) Matera, A. G., and Wang, Z. (2014) A day in the life of the spliceosome. Nat. Rev. Mol. Cell Biol. 15, 108-121.

(4) Nayler, O., Schnorrer, F., Stamm, S., and Ullrich, A. (1998) The cellular localization of the murine serine/arginine-rich protein kinase CLK2 is regulated by serine 141 autophosphorylation. J. Biol. Chem. 273, 34341-34348.

(5) Nam, S. Y., Seo, H. H., Park, H. S., An, S., Kim, J. Y., Yang, K. H., Kim, C. S., Jeong, M., and Jin, Y. W. (2010) Phosphorylation of CLK2 at serine 34 and threonine 127 by AKT controls cell survival after ionizing radiation. J. Biol. Chem. 285, 31157-31163.

(6) Rodgers, J. T., Haas, W., Gygi, S. P., and Puigserver, P. (2010) Cdc2-like kinase 2 is an insulin-regulated suppressor of hepatic gluconeogenesis. Cell Metab. 11, 23-34.

(7) Tabata, M., Rodgers, J. T., Hall, J. A., Lee, Y., Jedrychowski, M. P., Gygi, S. P., and Puigserver, P. (2014) Cdc2-like kinase 2 suppresses hepatic fatty acid oxidation and ketogenesis through disruption of the PGC-1alpha and MED1 complex. Diabetes 63, 1519-1532.

(8) Jain, P., Karthikeyan, C., Moorthy, N. S., Waiker, D. K., Jain, A. K., and Trivedi, P. (2014) Human CDC2-Like Kinase 1 (CLK1): A Novel Target for Alzheimer's Disease. Curr. Drug Targets 15, 539-550.

(9) Glatz, D. C., Rujescu, D., Tang, Y., Berendt, F. J., Hartmann, A. M., Faltraco, F., Rosenberg, C., Hulette, C., Jellinger, K., Hampel, H., Riederer, P., Moller, H. J., Andreadis, A., Henkel, K., and Stamm, S. (2006) The alternative splicing of tau exon 10 and its regulatory proteins CLK2 and TRA2-BETA1 changes in sporadic Alzheimer's disease. J. Neurochem. 96, 635-644.

(10) Winfield, S. L., Tayebi, N., Martin, B. M., Ginns, E. I., and Sidransky, E. (1997) Identification of three additional genes contiguous to the glucocerebrosidase locus on chromosome 1q21: implications for Gaucher disease. Genome research 7, 1020-1026.

(11) Nothwang, H. G., Kim, H. G., Aoki, J., Geisterfer, M., Kubart, S., Wegner, R. D., van Moers, A., Ashworth, L. K., Haaf, T., Bell, J., Arai, H., Tommerup, N., Ropers, H. H., and Wirth, J. (2001) Functional hemizygosity of PAFAH1B3 due to a PAFAH1B3-CLK2 fusion gene in a female with mental retardation, ataxia and atrophy of the brain. Human molecular genetics 10, 797-806.

(12) Wong, R., Balachandran, A., Mao, A. Y., Dobson, W., GrayOwen, S., and Cochrane, A. (2011) Differential effect of CLK SR Kinases on HIV-1 gene expression: potential novel targets for therapy. Retrovirology 8, 47.

(13) Karlas, A., Machuy, N., Shin, Y., Pleissner, K. P., Artarini, A., Heuer, D., Becker, D., Khalil, H., Ogilvie, L. A., Hess, S., Maurer, A. P., Muller, E., Wolff, T., Rudel, T., and Meyer, T. F. (2010) Genome-wide 
RNAi screen identifies human host factors crucial for influenza virus replication. Nature 463, 818-822.

(14) Fedorov, O., Huber, K., Eisenreich, A., Filippakopoulos, P., King, O., Bullock, A. N., Szklarczyk, D., Jensen, L. J., Fabbro, D., Trappe, J., Rauch, U., Bracher, F., and Knapp, S. (2011) Specific CLK inhibitors from a novel chemotype for regulation of alternative splicing. Chem. Biol. 18, 67-76.

(15) Hernandez-Lopez, H. R., and Graham, S. V. (2012) Alternative splicing in human tumour viruses: a therapeutic target? Biochem. J. 445, $145-156$.

(16) Gregori-Puigjane, E., and Mestres, J. (2008) A ligand-based approach to mining the chemogenomic space of drugs. Comb. Chem. High Throughput Screening 11, 669-676.

(17) Bain, J., Plater, L., Elliott, M., Shpiro, N., Hastie, C. J., McLauchlan, H., Klevernic, I., Arthur, J. S., Alessi, D. R., and Cohen, P. (2007) The selectivity of protein kinase inhibitors: a further update. Biochem. J. 408, 297-315.

(18) Jones, G., Willett, P., Glen, R. C., Leach, A. R., and Taylor, R. (1997) Development and validation of a genetic algorithm for flexible docking. J. Mol. Biol. 267, 727-748.

(19) Jones, G., Willett, P., and Glen, R. C. (1995) Molecular recognition of receptor sites using a genetic algorithm with a description of desolvation. J. Mol. Biol. 245, 43-53.

(20) Verdonk, M. L., Cole, J. C., Hartshorn, M. J., Murray, C. W., and Taylor, R. D. (2003) Improved protein-ligand docking using GOLD. Proteins: Struct., Funct., Genet. 52, 609-623.

(21) Muraki, M., Ohkawara, B., Hosoya, T., Onogi, H., Koizumi, J., Koizumi, T., Sumi, K., Yomoda, J., Murray, M. V., Kimura, H., Furuichi, K., Shibuya, H., Krainer, A. R., Suzuki, M., and Hagiwara, M. (2004) Manipulation of alternative splicing by a newly developed inhibitor of Clks. J. Biol. Chem. 279, 24246-24254.

(22) DeLano, W. L. (2002) The PyMol Molecular Graphics System, DeLano Scientific, Palo Alto, CA.

(23) Pogacic, V., Bullock, A. N., Fedorov, O., Filippakopoulos, P., Gasser, C., Biondi, A., Meyer-Monard, S., Knapp, S., and Schwaller, J. (2007) Structural analysis identifies imidazo[1,2-b]pyridazines as PIM kinase inhibitors with in vitro antileukemic activity. Cancer Res. 67, 6916-6924.

(24) Yamaguchi, H., Miwa, Y., Kasa, M., Kitano, K., Amano, M., Kaibuchi, K., and Hakoshima, T. (2006) Structural basis for induced-fit binding of Rho-kinase to the inhibitor Y-27632. J. Biochem. 140, 305311.

(25) Bullock, A. N., Das, S., Debreczeni, J. E., Rellos, P., Fedorov, O., Niesen, F. H., Guo, K., Papagrigoriou, E., Amos, A. L., Cho, S., Turk, B. E., Ghosh, G., and Knapp, S. (2009) Kinase domain insertions define distinct roles of CLK kinases in SR protein phosphorylation. Structure 17, 352-362.

(26) Mott, B. T., Tanega, C., Shen, M., Maloney, D. J., Shinn, P., Leister, W., Marugan, J. J., Inglese, J., Austin, C. P., Misteli, T., Auld, D. S., and Thomas, C. J. (2009) Evaluation of substituted 6arylquinazolin-4-amines as potent and selective inhibitors of cdc2like kinases (Clk). Bioorg. Med. Chem. Lett. 19, 6700-6705.

(27) Li, B., Pai, R., Aiello, D., Di, M., Barnes, M. H., Peet, N. P., Bowlin, T. L., and Moir, D. T. (2013) Optimization of a novel potent and selective bacterial DNA helicase inhibitor scaffold from a high throughput screening hit. Bioorg. Med. Chem. Lett. 23, 3481-3486.

(28) Bento, A. P., Gaulton, A., Hersey, A., Bellis, L. J., Chambers, J., Davies, M., Kruger, F. A., Light, Y., Mak, L., McGlinchey, S., Nowotka, M., Papadatos, G., Santos, R., and Overington, J. P. (2014) The ChEMBL bioactivity database: an update. Nucleic Acids Res. 42, D1083-1090.

(29) Urich, R., Wishart, G., Kiczun, M., Richters, A., Tidten-Luksch, N., Rauh, D., Sherborne, B., Wyatt, P. G., and Brenk, R. (2013) De novo design of protein kinase inhibitors by in silico identification of hinge region-binding fragments. ACS Chem. Biol. 8, 1044-1052.

(30) Boutros, M., Bras, L. P., and Huber, W. (2006) Analysis of cellbased RNAi screens. Genome biology 7, R66. 